\title{
Supplementary Material to
}

Tautomeric and Conformational Properties of Acetoacetamide, $\mathrm{CH}_{3} \mathrm{C}(\mathrm{O})-\mathrm{CH}_{2}-\mathrm{C}(\mathrm{O}) \mathrm{NH}_{2}$ : Electron Diffraction and Quantum Chemical Study.

\author{
Natalya V. Belova, ${ }^{\S}$ Heinz Oberhammer, ${ }^{\#}$ Georgiy V. Girichev,${ }^{\S}$ Sergey A. Shlykov. ${ }^{\S}$ \\ ${ }^{\S}$ Ivanovo State University of Chemistry and Technology, Ivanovo 153460, Russia \\ \# Institut für Physikalische und Theoretische Chemie, Universität Tübingen, 72076 Tübingen,
}

Germany

belova@isuct.ru

\section{Contents.}

\begin{tabular}{|l|c|}
\hline $\begin{array}{l}\text { Results of MP2 calculations with 6-31G(d,p) basis sets: cartesian coordinates, } \\
\text { electronic energies, number of imaginary frequencies, zero point corrections, } \\
\text { thermal corrections to Energies, Enthalpies and Gibbs Free Energies for enol and } \\
\text { diketo confomers. }\end{array}$ & $\mathrm{S} 2$ \\
\hline $\begin{array}{l}\text { Results of B3LYP calculations with 6-31G(d,p) basis sets: cartesian coordinates, } \\
\text { electronic energies, number of imaginary frequencies, zero point corrections, } \\
\text { thermal corrections to Energies, Enthalpies and Gibbs Free Energies for enol and } \\
\text { diketo confomers. }\end{array}$ & $\mathrm{S} 3$ \\
\hline $\begin{array}{l}\text { Results of MP2 calculations with 6-311++G(3df,pd) basis sets: cartesian } \\
\text { coordinates, electronic energies, number of imaginary frequencies, zero point } \\
\text { corrections, thermal corrections to Energies, Enthalpies and Gibbs Free Energies for } \\
\text { enol and diketo confomers. }\end{array}$ & $\mathrm{S} 4$ \\
\hline $\begin{array}{l}\text { Results of B3LYP calculations with 6-311++G(3df,pd) basis sets: cartesian } \\
\text { coordinates, electronic energies, number of imaginary frequencies, zero point } \\
\text { corrections, thermal corrections to Energies, Enthalpies and Gibbs Free Energies for } \\
\text { enol and diketo confomers. }\end{array}$ & $\mathrm{S} 5$ \\
\hline
\end{tabular}




\section{Structure of the enol form:}

\section{MP2/6-31G(d,p) geometry optimization}

\begin{tabular}{|c|c|c|c|c|c|}
\hline \multirow{2}{*}{$\begin{array}{l}\text { Center } \\
\text { Number }\end{array}$} & \multirow{2}{*}{$\begin{array}{l}\text { Atomic } \\
\text { Number }\end{array}$} & \multirow{2}{*}{$\begin{array}{l}\text { Atomic } \\
\text { Type }\end{array}$} & \multicolumn{3}{|c|}{ Coordinates (Angstroms) } \\
\hline & & & $\mathrm{X}$ & \multirow{2}{*}{$\begin{array}{c}Y \\
------1\end{array}$} & Z \\
\hline \multicolumn{5}{|c|}{ - - - - - - - - - - - - - - - - - - - - - - - - - - - - - - - - - - - - - - - - - - - - - - - - - - - } & \\
\hline 1 & 6 & 0 & -0.007786 & -0.758038 & -0.006634 \\
\hline 2 & 6 & 0 & -1.269225 & -0.030529 & -0.002643 \\
\hline 3 & 6 & 0 & 1.169698 & -0.075132 & 0.000602 \\
\hline 4 & 8 & 0 & -1.330807 & 1.220818 & -0.002182 \\
\hline 5 & 8 & 0 & 1.240145 & 1.262367 & 0.008598 \\
\hline 6 & 7 & 0 & -2.415073 & -0.772196 & -0.038583 \\
\hline 7 & 6 & 0 & 2.512994 & -0.726806 & 0.000150 \\
\hline 8 & 1 & 0 & 0.292453 & 1.572812 & 0.002151 \\
\hline 9 & 1 & 0 & 0.003903 & -1.837182 & -0.022552 \\
\hline 10 & 1 & 0 & -2.395234 & -1.751719 & 0.182058 \\
\hline 11 & 1 & 0 & -3.276488 & -0.273041 & 0.107909 \\
\hline 12 & 1 & 0 & 2.427201 & -1.810243 & -0.004484 \\
\hline 13 & 1 & 0 & 3.070996 & -0.412494 & 0.881321 \\
\hline 14 & 1 & 0 & 3.073899 & -0.405215 & -0.876500 \\
\hline \multicolumn{6}{|c|}{ - - - - - - - - - - - - - - - - - - - - - - - - - - - - - - - - - - - - - - - - - - - - - - - - - - - - } \\
\hline \multirow{2}{*}{\multicolumn{6}{|c|}{$\begin{array}{l}\mathrm{E}(\mathrm{MP} 2)=-360.8232099 \mathrm{~A} . \mathrm{U} . \\
\text { No. of imaginary frequencies: } 0\end{array}$}} \\
\hline No. of & & lencies: 0 & & & \\
\hline \multicolumn{4}{|c|}{ Zero-point correction $=$} & 0.115432 & \multirow[t]{2}{*}{ (Hartree/Particle) } \\
\hline \multirow{2}{*}{\multicolumn{4}{|c|}{$\begin{array}{l}\text { Thermal correction to Energy= } \\
\text { Thermal correction to Enthalpy= }\end{array}$}} & 0.122934 & \\
\hline & & & & 0.123878 & \\
\hline Therma] & correction & Gibbs Free & ergy= & 0.084004 & \\
\hline
\end{tabular}

\section{Structure of the diketo form:}

\section{MP2/6-31G(d,p) geometry optimization}

\begin{tabular}{|c|c|c|c|c|c|}
\hline \multirow{2}{*}{$\begin{array}{l}\text { Center } \\
\text { Number }\end{array}$} & \multirow{2}{*}{$\begin{array}{l}\text { Atomic } \\
\text { Number }\end{array}$} & \multirow{2}{*}{$\begin{array}{l}\text { Atomic } \\
\text { Type }\end{array}$} & \multicolumn{3}{|c|}{ Coordinates (Angstroms) } \\
\hline & & & $\mathrm{X}$ & $\mathrm{Y}$ & Z \\
\hline & & & $----\cdots$ & -------- & ------- \\
\hline 1 & 6 & 0 & 0.003629 & -0.583276 & 0.670763 \\
\hline 2 & 6 & 0 & -1.334860 & -0.222850 & 0.023937 \\
\hline 3 & 6 & 0 & 1.205660 & 0.136315 & 0.077896 \\
\hline 4 & 8 & 0 & -2.116479 & -1.091068 & -0.357406 \\
\hline 5 & 8 & 0 & 1.214080 & 1.359354 & -0.062701 \\
\hline 6 & 7 & 0 & -1.598683 & 1.114348 & -0.012631 \\
\hline 7 & 6 & 0 & 2.386520 & -0.715290 & -0.305083 \\
\hline 8 & 1 & 0 & 0.111005 & -1.662698 & 0.602640 \\
\hline 9 & 1 & 0 & -0.044393 & -0.306731 & 1.727743 \\
\hline 10 & 1 & 0 & -0.817585 & 1.750830 & 0.049982 \\
\hline 11 & 1 & 0 & -2.406470 & 1.389722 & -0.547525 \\
\hline 12 & 1 & 0 & 3.193238 & -0.086353 & -0.669229 \\
\hline 13 & 1 & 0 & 2.087437 & -1.426659 & -1.075839 \\
\hline 14 & 1 & 0 & 2.721043 & -1.294229 & 0.556418 \\
\hline
\end{tabular}

$E(M P 2)=-360.8264223$ A.U.

No. of imaginary frequencies: 0

Zero-point correction

Thermal correction to Energy=

Thermal correction to Enthalpy=

0.115115 (Hartree/Particle)

0.122766

Thermal correction to Gibbs Free Energy=

0.123711

0.082406 
Structure of the enol form:

\section{B3LYP/6-31G(d,p) geometry optimization}

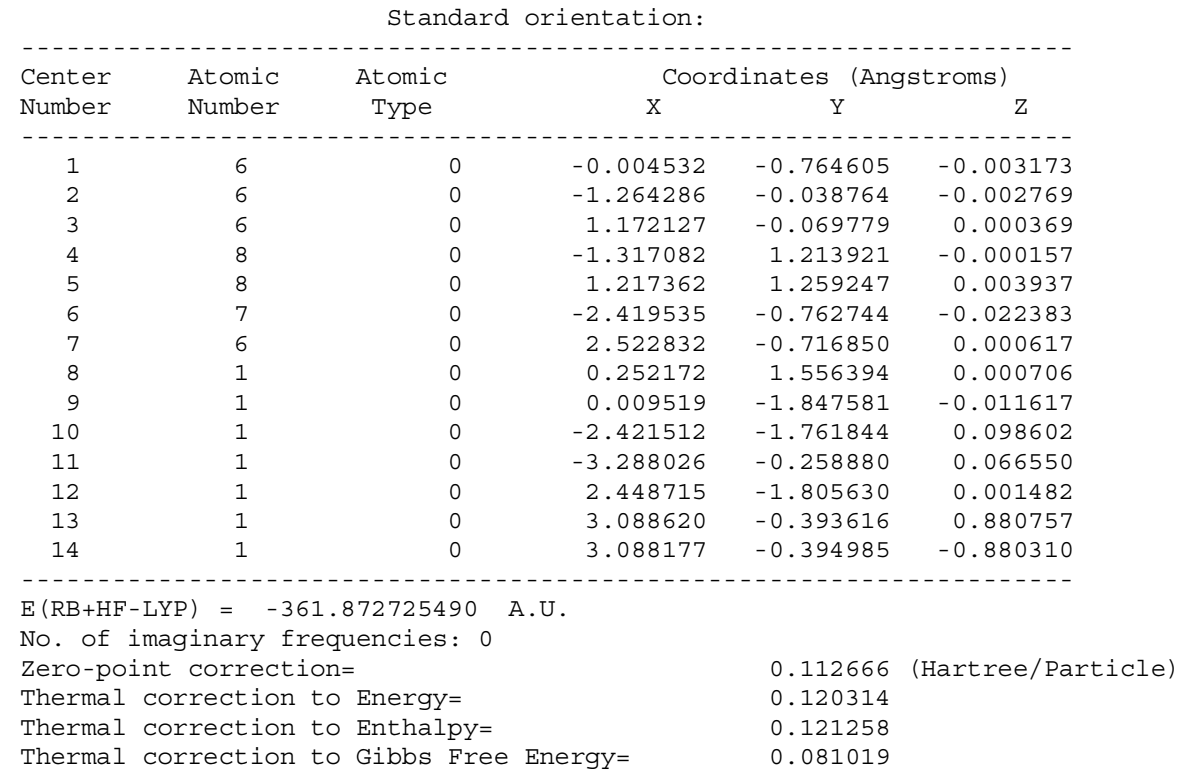

\section{Structure of the diketo form:}

\section{B3LYP/6-31G(d,p) geometry optimization}

\begin{tabular}{|c|c|c|c|c|c|}
\hline \multirow{2}{*}{$\begin{array}{l}\text { Center } \\
\text { Number }\end{array}$} & \multirow{2}{*}{$\begin{array}{l}\text { Atomic } \\
\text { Number }\end{array}$} & \multirow{2}{*}{$\begin{array}{l}\text { Atomic } \\
\text { Type }\end{array}$} & \multicolumn{3}{|c|}{ Coordinates (Angstroms) } \\
\hline & & & $\mathrm{X}$ & $\mathrm{Y}$ & Z \\
\hline & & & $----\cdots$ & -------- & ------- \\
\hline 1 & 6 & 0 & 0.007161 & -0.674624 & 0.511136 \\
\hline 2 & 6 & 0 & -1.374454 & -0.216309 & 0.009987 \\
\hline 3 & 6 & 0 & 1.223139 & 0.126546 & 0.055852 \\
\hline 4 & 8 & 0 & -2.220866 & -1.038097 & -0.307506 \\
\hline 5 & 8 & 0 & 1.198926 & 1.343431 & -0.046231 \\
\hline 6 & 7 & 0 & -1.579578 & 1.127700 & 0.028604 \\
\hline 7 & 6 & 0 & 2.477749 & -0.664089 & -0.240124 \\
\hline 8 & 1 & 0 & 0.106067 & -1.730598 & 0.254089 \\
\hline 9 & 1 & 0 & -0.006463 & -0.613433 & 1.609248 \\
\hline 10 & 1 & 0 & -0.771482 & 1.738215 & 0.075998 \\
\hline 11 & 1 & 0 & -2.439024 & 1.460943 & -0.382969 \\
\hline 12 & 1 & 0 & 3.309230 & 0.012940 & -0.438773 \\
\hline 13 & 1 & 0 & 2.307148 & -1.310836 & -1.108988 \\
\hline 14 & 1 & 0 & 2.725516 & -1.322947 & 0.599955 \\
\hline
\end{tabular}

$\mathrm{E}(\mathrm{RB}+\mathrm{HF}-\mathrm{LYP})=-361.869812425 \mathrm{~A} . \mathrm{U}$.

No. of imaginary frequencies: 0

Zero-point correction

Thermal correction to Energy=

Thermal correction to Enthalpy=

0.112306 (Hartree/Particle)

0.120060

Thermal correction to Gibbs Free Energy=

0.121004

0.079620 
Structure of the enol form:

\section{MP2/6-311++G(3df,pd) geometry optimization}

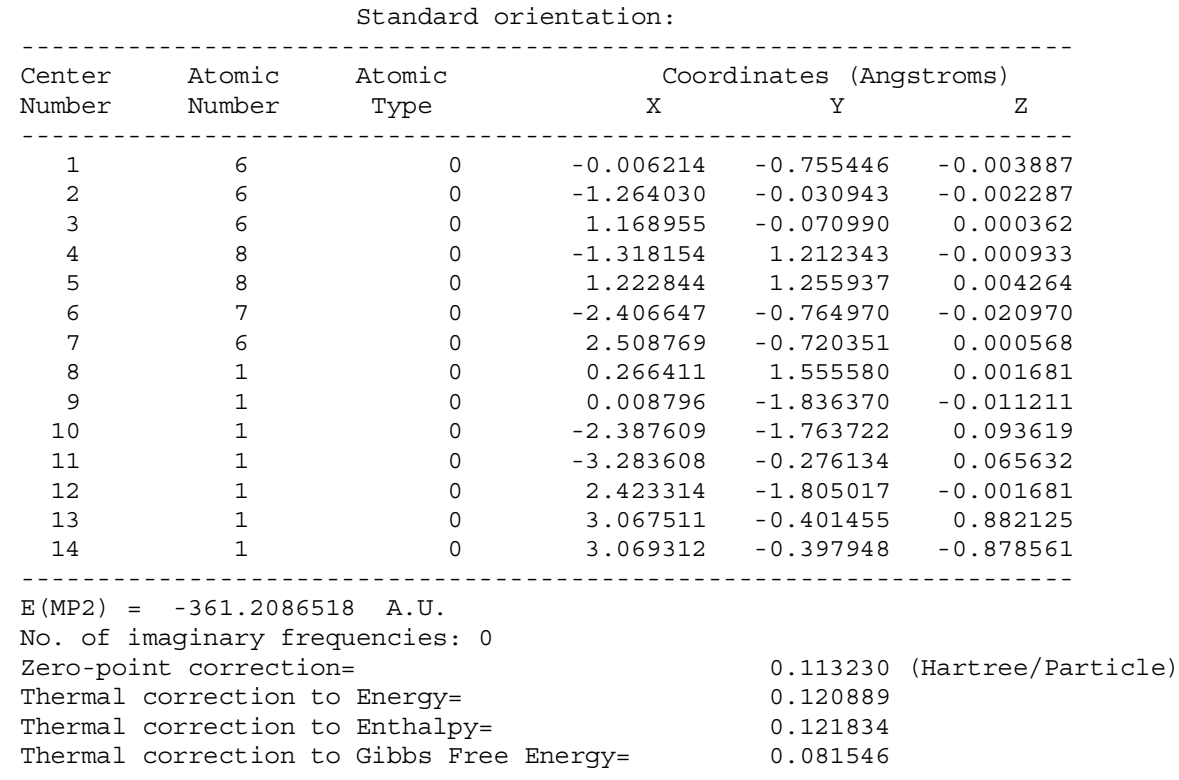

Structure of the diketo form:

MP2/6-311++G(3df,pd) geometry optimization

\begin{tabular}{|c|c|c|c|c|c|}
\hline \multirow{2}{*}{$\begin{array}{l}\text { Center } \\
\text { Number }\end{array}$} & \multirow{2}{*}{$\begin{array}{l}\text { Atomic } \\
\text { Number }\end{array}$} & \multirow{2}{*}{$\begin{array}{c}\text { Atomic } \\
\text { Type }\end{array}$} & \multicolumn{3}{|c|}{ Coordinates (Angstroms) } \\
\hline & & & $\mathrm{X}$ & $\mathrm{Y}$ & $\mathrm{Z}$ \\
\hline & & & ---- & ------- & -------- \\
\hline 1 & 6 & 0 & -0.011492 & -0.476036 & 0.807606 \\
\hline 2 & 6 & 0 & -1.303529 & -0.200738 & 0.039920 \\
\hline 3 & 6 & 0 & 1.197842 & 0.137144 & 0.126102 \\
\hline 4 & 8 & 0 & -2.053722 & -1.101354 & -0.295282 \\
\hline 5 & 8 & 0 & 1.306636 & 1. 347128 & 0.022391 \\
\hline 6 & 7 & 0 & -1.548872 & 1.114559 & -0.185576 \\
\hline 7 & 6 & 0 & 2.217704 & -0.815189 & -0.426046 \\
\hline 8 & 1 & 0 & 0.078974 & -1.554500 & 0.914318 \\
\hline 9 & 1 & 0 & -0.099341 & -0.015744 & 1.795675 \\
\hline 10 & 1 & 0 & -0.798835 & 1.782380 & -0.068985 \\
\hline 11 & 1 & 0 & -2.332252 & 1.345549 & -0.777498 \\
\hline 12 & 1 & 0 & 3.021650 & -0.271412 & -0.916105 \\
\hline 13 & 1 & 0 & 1.730395 & -1.491729 & -1.132990 \\
\hline 14 & 1 & 0 & 2.615052 & -1.433741 & 0.382245 \\
\hline
\end{tabular}

$E($ MP2 $)=-361.2060445$ A.U.

No. of imaginary frequencies: 0

Zero-point correction=

0.112696 (Hartree/Particle)

Thermal correction to Energy=
Thermal correction to Enthalpy=

0.120549

0.121493

Thermal correction to Gibbs Free Energy=

0.079309 
Structure of the enol form:

\section{B3LYP/6-311++G(3df,pd) geometry optimization}

\begin{tabular}{|c|c|c|c|c|c|}
\hline \multirow{2}{*}{$\begin{array}{l}\text { Center } \\
\text { Number }\end{array}$} & \multirow{2}{*}{$\begin{array}{l}\text { Atomic } \\
\text { Number }\end{array}$} & \multirow{2}{*}{$\begin{array}{l}\text { Atomic } \\
\text { Type }\end{array}$} & \multicolumn{3}{|c|}{ Coordinates (Angstroms) } \\
\hline & & & $\mathrm{X}$ & $\mathrm{Y}$ & $\mathrm{Z}$ \\
\hline \multicolumn{6}{|c|}{ 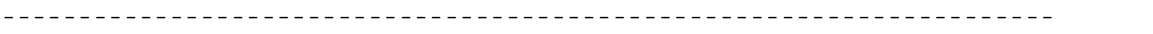 } \\
\hline 1 & 6 & 0 & 0.000000 & 0.751543 & 0.000000 \\
\hline 2 & 6 & 0 & 1.265035 & 0.040650 & 0.000000 \\
\hline 3 & 6 & 0 & -1.172330 & 0.062376 & 0.000000 \\
\hline 4 & 8 & 0 & 1.330108 & -1.202534 & 0.000000 \\
\hline 5 & 8 & 0 & -1.217134 & -1.263534 & 0.000000 \\
\hline 6 & 7 & 0 & 2.406500 & 0.778726 & 0.000000 \\
\hline 7 & 6 & 0 & -2.517517 & 0.707738 & 0.000000 \\
\hline 8 & 1 & 0 & -0.263232 & -1.573500 & 0.000000 \\
\hline 9 & 1 & 0 & -0.018003 & 1.831550 & 0.000000 \\
\hline 10 & 1 & 0 & 2.393676 & 1.783059 & 0.000000 \\
\hline 11 & 1 & 0 & 3.289781 & 0.296433 & 0.000000 \\
\hline 12 & 1 & 0 & -2.440790 & 1.793045 & 0.000000 \\
\hline 13 & 1 & 0 & -3.080928 & 0.386514 & 0.878967 \\
\hline 14 & 1 & 0 & -3.080928 & 0.386514 & -0.878967 \\
\hline \multicolumn{6}{|c|}{ - - - - - - - - - - - - - - - - - - - - - - - - - - - - - - - - - - - - - - - - - - - - - - - - - - - - } \\
\hline \multirow{2}{*}{\multicolumn{6}{|c|}{$\begin{array}{l}\mathrm{E}(\mathrm{RB}+\mathrm{HF}-\mathrm{LYP})=-362.003593562 \mathrm{~A} . \mathrm{U} . \\
\text { No. of imaginary frequencies: } 0\end{array}$}} \\
\hline & & & & & \\
\hline \multicolumn{4}{|c|}{ Zero-point correction = } & 0.112044 & (Hartree/Particle) \\
\hline \multicolumn{4}{|c|}{ Thermal correction to Energy= } & 0.119830 & \\
\hline \multicolumn{4}{|c|}{ Thermal correction to Enthalpy= } & 0.120775 & \\
\hline Thermal & orrectior & Gibbs Free & ergy= & 0.079935 & \\
\hline
\end{tabular}

Structure of the diketo form:

\section{B3LYP/6-311++G(3df,pd) geometry optimization}

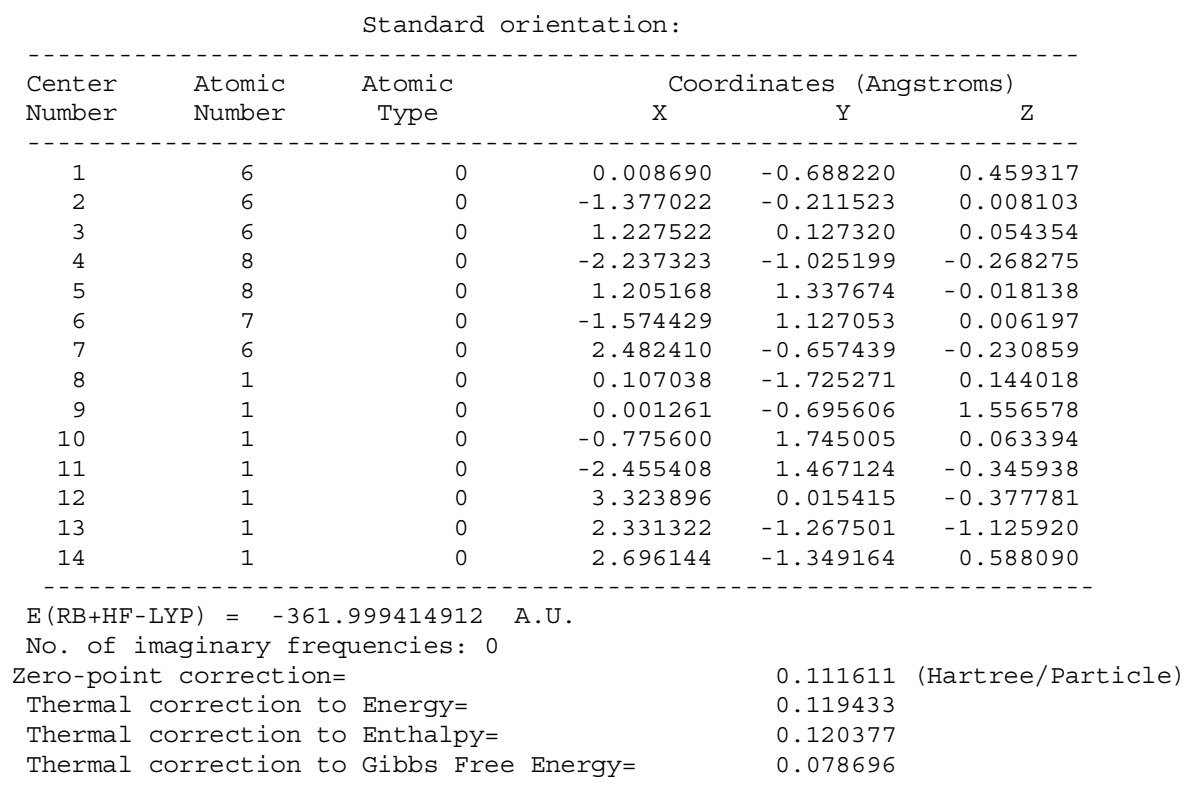

